\title{
LA EXPORTACIÓN DE TURISMO, UN PILAR PARA EL DESARROLLO ECONÓMICO DE LA PROVINCIA DE GUANACASTE EN COSTA RICA
}

Héctor Canossa Montes de Oca, MSc.

https://orcid.org/0000-0003-0952-3193

Máster en Administración de Negocios con énfasis en Mercadeo (Costa Rica).

Docente Tiempo Completo de la Universidad Técnica Nacional, Costa Rica. Docente a Tiempo Parcial de la Universidad Nacional de Costa Rica, Costa Rica. hcanossa@utn.ac.cr

\section{ARTÍCULO DE REFLEXIÓN}

Recibido: 14 de enero 2020.

Aceptado: 23 de marzo de 2020.

\section{RESUMEN}

El propósito de este estudio es analizar el turismo como un pilar del desarrollo económico de Guanacaste, provincia del noroeste de Costa Rica donde se requieren esfuerzos para evaluar su potencial e importancia para el país, por consiguiente, los objetivos específicos incluyen describir la situación actual de la exportación de turismo en esta zona, mencionar expectativas del turismo en Guanacaste y reconocer desafíos del sector. Se hace un estudio exploratorio a través de una revisión literaria identificando que para lograr desarrollo implica retos en infraestructura y servicios como carreteras, fortalecimiento de la educación en las áreas de servicio e idioma, además se deben buscar vínculos productivos, buscando promover Guanacaste como un sector para el futuro de Costa Rica, donde el turismo es generador de empleo y bienestar para la población, por lo que se deben generar condiciones óptimas para atraer más turistas e inversionistas enriqueciendo las ventajas competitivas. 
Palabra clave: bienestar, exportación de servicios, impacto económico del turismo, desarrollo turístico, Costa Rica.

\section{ABSTRACT}

The purpose of this study is to analyze tourism as a pillar of the economic development of Guanacaste, province of northwestern Costa Rica, where efforts can be made to assess its potential and importance for the country, the specific objectives include describe the current situation of tourism exports in this area, mention tourism expectations in Guanacaste and recognize challenges of the sector. An exploratory study is carried out through a literary review identifying that to achieve the development involved challenges in infrastructure and services such as roads, strengthening education in the areas of service and language, in addition productive links should be sought, seeking to promote Guanacaste as a sector for the future of Costa Rica, where tourism is a generator of employment and welfare for the population, so they must generate optimal conditions to attract more tourists and investors enriching the competitive advantages.

Keywords: welfare, export of services, economic impact of tourism, tourism development, Costa Rica.

\section{INTRODUCCIÓN}

El turismo puede promover el desarrollo económico de un país, ya que este sector genera inversiones en forma de infraestructura y oferta de servicios Por lo tanto, genera fuentes de empleo tanto directo como indirecto; donde se tienen las expectativas de condiciones óptimas que permitan a las personas tener un mayor bienestar y calidad de vida, así como oportunidades de negociación para los productores locales. Por esto, se considera relevante el estudio ya que el turismo puede contribuir mucho a la provincia de Guanacaste, Costa Rica y así verse también como un caso de estudio para otras regiones o países. Una provincia como Guanacaste en Costa Rica, por su posición geográfica y gran riqueza ecológica, es clave para comercializar servicios turísticos a nivel global, un país sin ejército, con capital humano que tiene múltiples oportunidades 
educativas, adquiere la posibilidad de explotar ventajas competitivas que están disponibles actualmente.

Entre las ventajas de la exportación de turismo, se encuentra generar empleo en el sector servicios con salarios más altos comparativamente con otros sectores y por lo tanto mayor posibilidad de calidad de vida. Por otro lado, esta exportación a medida que el sector crece en Costa Rica genera un pago de impuestos que pueden contribuir al presupuesto nacional y cumplir algunos deseos de muchos costarricenses, como la mejora en la infraestructura vial. Como desventajas, se puede mencionar que a pesar de promover el ecoturismo y ser una de las principales ventajas competitivas en Costa Rica a nivel mundial, este aumento en la infraestructura siempre genera impacto ambiental, además, los propios turistas al ingresar a estas áreas también generan impacto, por ejemplo, con el sonido y los colores que tienen efectos sobre la vida silvestre, por lo que se deben definir estrategias para la mitigación, reducción o prevención de estos impactos.

Entonces, para que este turismo sea un pilar óptimo de desarrollo debera ser turismo de calidad, satisfaciendo necesidades y expectativas, así como entregando beneficios justos a las partes involucradas y promoviendo la sostenibilidad, como expone la World Tourism Organization (2017), en el contexto del turismo, la calidad no consiste solo en producir "cero defectos" y cumplir requisitos básicos; también significa cuidado, innovación y excelencia; no es simplemente una cuestión de satisfacer las expectativas de los clientes sino superarlas. Para esto se necesita un liderazgo comprometido de los empresarios y fomentar el compromiso y coordinación entre, las entidades públicas y privadas interesadas (p. 13).

Por consiguiente, el objetivo general de este trabajo es analizar el turismo como un pilar del desarrollo económico de Guanacaste y como objetivos específicos se busca describir la situación actual de la exportación de servicios turísticos en el país, mencionar las expectativas de turismo en Guanacaste para los próximos años y reconocer desafíos para que la exportación de turismo sea un generador de desarrollo económico. Metodológicamente, se considera un estudio exploratorio teniendo por propósito examinar un tema de investigación poco estudiado o que no se ha abordado amplamente 
con anterioridad, esto pretende motivar futuras investigaciones (Hernández, Méndez, Mendoza y Cuevas, 2017, p. 75).

\section{REVISIÓN TEÓRICA}

\section{Situación actual de la exportación de servicios turísticos}

En las últimas seis décadas, el turismo ha experimentado una continua expansión y diversificación, convirtiéndose en uno de los sectores económicos más grandes y de más rápido crecimiento en el mundo. La expansión del sector de servicios, la mayor competencia resultante de la globalización y auge de tecnologías de la información han acelerado el cambio hacia una economía basada en el conocimiento y la innovación. Para poder desarrollar turismo de calidad, las organizaciones necesitan saber cuál es su ventaja competitiva y qué capacidades necesitan para crecer y mantenerse (Ţîtu, Răulea y Tुîtu 2016, pp. 294-295).

En el mundo y los países el turismo representa una importante fuente de ingresos, ya que para las personas realizar actividades turísticas representa una necesidad, por esto el crecimiento tan amplio y la representación de estos servicios en las producciones nacionales y en la exportación de los servicios. Como expone Palafox Muñoz (2013):

El paisaje integrado por los recursos naturales y culturales son de interés para la economía global al ser considerados la materia prima para la planeación, organización, desarrollo y comercialización del turismo, aunado a la necesidad del modo de producción capitalista por transformarse y revitalizarse. Para ello, los organismos internacionales reconocen que el turismo es un medio para la consolidación del modelo cultural vinculado al modelo económico, así la generación de divisas, el impulso a la inversión privada y crear un nuevo producto de exportación sin la necesidad de costos de producción, aunado a la necesidad de los Estados subdesarrollados por la creación de empleos y combatir la 
pobreza, hacen que la actividad turística se constituya en un eje de acumulación (p. 162).

En los siguientes párrados se identifican las ventajas que tiene Costa Rica, en el mercado turístico considerando sus principales atractivos, el comportamiento de las entradas de turistas en el país, así como la contribución del turismo en la economía costarricense.

\section{Ventajas competitivas de Guanacaste y Costa Rica para el mercado turístico internacional}

Costa Rica tiene ventajas competitivas en el mercado turístico internacional, como la biodiversidad del país, lo que hace del país un exponente del ecoturismo y en Guanacaste se puede ejemplificar con la contribución que brinda el Área de Conservación de Guanacaste, definido como un Patrimonio de la Humanidad, tiene una área de vida silvestre protegida de 163,000 hectáreas y aproximadamente 335,000 especies de organismos terrestres, lo que representaría el $2.6 \%$ de la biodiversidad del mundo (Área de Conservación de Guanacaste, 2019).

Como otras ventajas competitivas para las empresas internacionales que involucran el turismo son la ubicación geográfica, el acceso al Océano Pacífico y el Atlántico y la gran cantidad de playas disponibles en Guanacaste. Además, el país al no tener un ejército es otro punto a favor, considerando que los inversores con esto, pueden percibir una mayor estabilidad política, lo que influiría positivamente en sus decisiones en comparación con otros países. Asimismo, se están haciendo esfuerzos para promover la sostenibilidad y el medio ambiente, que son cuestiones clave para generar una ventaja competitiva a nivel internacional, por lo que vale la pena mencionar los programas de Certificación de Sostenibilidad Turística (CST) y el Programa de Bandera Azul Ecológica (BAE), en el que actualmente hay al menos 235 hoteles (41 en Guanacaste), 6 empresas gastronómicas, 16 parques temáticos y 84 agencias de tour operadores (5 en Guanacaste) (Instituto Costarricense de Turismo, 2019). 
Ventura, citado por la Presidencia de la República de Costa Rica (2016) expone que "el turismo es sinónimo de progreso, bienestar, crecimiento socioeconómico, protección del medio ambiente y una plataforma ideal para la promoción del patrimonio cultural. Además, gracias a nuestro Modelo de Desarrollo de Turismo Sostenible, esta actividad se ha convertido en la mejor herramienta para generar empleo y progreso social y económico en las comunidades".

Para finalizar este apartado, es importante mencionar algunos atractivos que tiene Guanacaste y que es relevante continuar estudiando y desarrollando para fortalecer su atractivo, en el canton de La Cruz, Guanacaste se encuentra Área Marina de Manejo Bahía Santa Elena, además de la antes mencionada Área de Conservación Guanacaste. En el cantón de Bagaces, hay una diversidad de oferta de servicios ligados a aguas termales; por su parte, en el cantón de Tilaran, se ubican sitios como parte del lago Arenal y acceso a Cerro Pelado, además, en Guanacaste también se tiene el Polo Turístico Golfo de Papagayo, con una gran oferta de hoteles de playa. En fin, estos son algunos ejemplos de atractivos, y no se puede dejar de lado los aspectos de turismo rural, ecológico y cultural que permiten un mayor aprovechamiento del turismo.

\section{Comportamiento de la entrada de turistas extranjeros a Costa Rica}

El atractivo y las ventajas del país y de Guanacaste se reflejan con números, según el Instituto Costarricense de Turismo (2019), durante 2016 Costa Rica superó los 2,9 millones de llegadas internacionales y de estos, el Aeropuerto Internacional Daniel Oduber de Liberia en Guanacaste recibió 572.544 turistas. En este aeropuerto, se han superado más de 5 millones de pasajeros en los últimos cinco años, debido a factores como el aumento en el número de aerolíneas presentes en la terminal (Jiménez, 2017).

Es notable cómo se muestra en la tabla 1, que la llegada de turistas tiene un aumento constante, solo el año 2009 tuvo una disminución debido a los efectos de la crisis financiera de 2008, pero en 2018, hubo más de tres millones de llegadas internacionales, este dato para el país es relevante considerando que tiene una población de cinco millones de habitantes. 
Tabla 1. Comportamiento de actividad turística en Costa Rica entre 2013 y 2018.

\begin{tabular}{|c|c|c|c|c|c|c|}
\hline $\begin{array}{l}\text { Información } \\
\text { relevante }\end{array}$ & 2013 & 2014 & 2015 & 2016 & 2017 & 2018 \\
\hline $\begin{array}{l}\text { Llegadas } \\
\text { internacionales }\end{array}$ & 2427941 & $\begin{array}{c}2526 \\
817\end{array}$ & 2660257 & 2925128 & $\begin{array}{c}2959 \\
869\end{array}$ & $\begin{array}{c}3016 \\
667\end{array}$ \\
\hline $\begin{array}{l}\text { Gasto } \\
\text { promedio en } \\
\text { dólares }(\$)\end{array}$ & 1322,6 & 1351,3 & 1369,3 & 1309,1 & 1561,4 & 1335,4 \\
\hline $\begin{array}{l}\text { Estancia } \\
\text { media en } \\
\text { noches }\end{array}$ & 11,6 & 12,4 & 11,3 & 12,1 & 11,9 & 11,7 \\
\hline $\begin{array}{l}\text { Personas que } \\
\text { trabajan en } \\
\text { hoteles y } \\
\text { restaurantes. }\end{array}$ & 58646 & 59444 & 61795 & 65239 & 67311 & 69563 \\
\hline $\begin{array}{l}\text { Cantidad de } \\
\text { habitaciones } \\
\text { disponibles } \\
\text { para } \\
\text { hospedaje }\end{array}$ & 46633 & 46375 & 46819 & 47051 & 56334 & 57233 \\
\hline
\end{tabular}

Notas: La tabla muestra el comportamiento del turismo en Costa Rica, de acuerdo con los datos estadísticos obtenidos del Instituto Costarricense de Turismo, los datos relevantes incluyen las llegadas internacionales, el promedio de gastos en dólares estadounidenses, el promedio de estadías en noches, el número de personas trabajando en hoteles y restaurantes y la cantidad de habitaciones disponibles para el alojamiento, esto desde el año 2013 hasta el año 2018, alrededor del año 2019 todavía no hay suficientes estadísticas.

Fuente: Elaboración propia con base en el Instituto Costarricense de Turismo, 2019. 
Cabe señalar que según el Instituto Costarricense de Turismo (2019), la estadía promedio del turista es de aproximadamente 12 noches y el costo promedio por persona es de alrededor de $\$ 1.300$ durante la estadía. Además, en relación con Guanacaste, se debe agregar que las entradas del Aeropuerto Internacional Daniel Oduber también han tenido un aumento constante, incluso destacando que entre 2015 y 2016 el aumento correspondió al 34\%, de 426,336 llegadas a 572,544 llegadas. En el año 2018 en este aeropuerto de Guanacaste las llegadas fueron de 552,589 y durante el primer semestre de 2019 hubo 310,118 visitantes y de estos $75 \%$, viajan por vacaciones y $68 \%$ disfrutan del sol y las playas de Costa Rica (Instituto Costarricense de Turismo, 2019).

\section{Contribución del turismo a la economía costarricense.}

La industria del turismo aporta el 6,3\% del PIB a la economía de Costa Rica (Instituto Costarricense de Turismo, 2019). Esto muestra la relevancia del sector para la economía costarricense, con un crecimiento constante a excepción de 2009 debido a los efectos de la crisis financiera global, incluso las exportaciones de servicios representaron el $55 \%$ de las exportaciones totales del país.

Por otro lado, refiriéndose al impacto económico del sector para Costa Rica, Segura (2017) cita que los datos proporcionados por el Instituto Costarricense de Turismo revelan que el turismo genera empleo para 600,000 personas que trabajan, directa o indirectamente, casi el $27 \%$ de la fuerza laboral del país. Además, considerando solo el empleo directo, el sector genera más del $6 \%$ del empleo a nivel nacional, esto considerando solo las actividades de vivienda y servicio de alimentos, alcanzando una cantidad de 158,772 empleos directos de la población empleada (Instituto Nacional de Estadísticas y Censos citados por el Instituto Costarricense de Turismo, 2019), esto sin considerar los servicios de transporte y la operación de recorridos terrestres y marítimos, servicios aéreos, orientación y empleo en áreas protegidas.

\section{Expectativas del turismo en Guanacaste}

\section{Crecimiento de proyectos turísticos en la zona}


Considernando la inversión extranjera y negocios internacionales, los nuevos proyectos de recreación y hotelería, generan altas expectativas sobre el progreso en infraestructura, generación de empleo y desarrollo económico. A nivel de país, en los hoteles, la tendencia de crecimiento se centra en Guanacaste con más de 50 mil metros en permisos procesados, es decir, más de cinco veces los procesados en áreas como la capital San José. Además, la oferta de habitaciones de hotel en Costa Rica es más de 50.000, según el Instituto Costarricense de Turismo (citado por Barquero, 2017). Esto proporciona indicaciones claras del aumento de la demanda de este tipo de servicios y su potencial. Además, está claro el aumento en la construcción de hoteles en el área, según lo indicado por Barquero (2017) "la inversión en nuevos hoteles con declaración de interés turístico por parte del Instituto Costarricense de Turismo ascenderá a \$149 millones en 2017".

\section{Requisitos de infraestructura}

Es evidente que para enfrentar este aumento en la demanda de turismo es necesario crecer en infraestructura, tal como servicios básicos, agua, electricidad y comunicaciones, donde debe resaltarse una situación para analizar en detalle, que es el acceso al agua potable. Además, la gran relevancia del acceso por carreteras y los traslados eficientes. Si la infraestructura vial no cumple con las expectativas de los turistas, esto puede causar que un porcentaje no visite Costa Rica o no repita la visita, también puede hacer que los turistas decidan venir al país, pero que se encuentren en un solo lugar o pocos debido a la dificultad en los traslados, que afecta los ingresos económicos de diversos lugares.

Actualmente, ciudades como Liberia se han convertido en áreas de suministro. Esto se evidencia por el auge de grandes supermercados en la provincia, ejemplificado por WalMart y su nueva ubicación en Liberia, Guanacaste, así como también la apertura de Pequeño Mundo y para el 2020 de Pricesmart. Sobre esto, Fallas (2017) explica que WalMart comenzó su incursión de la marca fuera del Gran Área Metropolitana y que se unirá a una lista de más de 50 supermercados que mantienen operaciones en diferentes áreas de Guanacaste, una mayor cantidad de las existentes en Heredia, Cartago y Puntarenas. 
En resumen, se considera que Guanacaste necesita mejorar la infraestructura vial, por ejemplo, la carretera alternativa al Aeropuerto Internacional, extensión de carriles en la ruta 550 de Liberia-Nicoya, mejoramiento de carreteras a las playas. Además, que se tengan planes alternativos para el suministro de agua. También, se supone que estas inversiones pueden realizarse y serían factibles ya que los ingresos del turismo y la generación de impuestos darían el rendimiento de las inversiones, aunque está claro que se necesita una gestión efectiva dentro del gobierno y cada municipalidad involucrada.

\section{Desafíos para la exportación del turismo para ser un generador de desarrollo económico: influencia de la educación para la optimización del empleo en el sector turístico}

Uno de los desafíos para que la exportación de turismo sea efectiva en la generación de desarrollo económico es la necesidad de capital humano de calidad, por lo que la educación es un buen punto de partida. Esto es importante porque si no se promueve aun más esta área, la contratación de empleados de turismo seguirá concentrándose en áreas operativas como servicio al cliente el mantenimiento, y para los puestos gerenciales se traerá capital humano de otras áreas del país o incluso de otros países.

En Guanacaste, instituciones como la Universidad de Costa Rica, Universidad Técnica Nacional, Universidad Nacional y el Instituto Nacional de Aprendizaje tienen el turismo dentro de su oferta académica, por lo que la población del área tiene la posibilidad de obtener un título técnico o universtario sin salir de Guanacaste. En este aspecto cabe mencionar la relevancia de las relaciones entre las intituciones educativas y el sector productivo turismo, para ajustar las ofertas académicas a la realidad de la demanda laboral. Así también, como punto clave, la educación turística debe combinarse con un segundo idioma, principalmente inglés, ya que la mayoría de los turistas que visitan Costa Rica provienen de los Estados Unidos. Además, hablar inglés parece que continúa siendo una debilidad de Guanacaste, y las personas deben enfrentarse a esto para mejorar sus oportunidades dentro del sector turístico. 
Los empleadores continúan quejándose del bajo nivel de idioma que traen los jóvenes. El informe del proyecto Combatiendo la Pobreza a través del empleo en Guanacaste, de la asociación Horizonte Positivo para la Segunda Vicepresidencia de la República, señala que el $95 \%$ de la población mayor de edad en la provincia no habla un segundo idioma (La Voz de Guanacaste, 2017).

\section{Importancia de los vínculos productivos para una mejor distribución del ingreso.}

Los turistas, viéndolos como clientes o usuarios de productos y servicios en Costa Rica, pueden tener un gran porcentaje de comunicación con las agencias de viajes y tener un itinerario establecido, por lo que es importante buscar alternativas para poder visitar diferentes lugares y, por lo tanto, más empresas puedan beneficiarse y obtener ingresos del turismo. Además, dentro de los hoteles y empresas ya establecidas en el sector, es importante promover los vínculos productivos y el enfoque en los productores del área local. Por ejemplo, que un gran hotel en lugar de comprar grandes proveedores de verduras y frutas, tenga apertura para comprar en el mercado local a pequeños productores.

Para lograr estos vínculos es un desafío que, en el caso de las pymes y los pequeños productores, cumplan con los estándares de calidad solicitados, además de cubrir la demanda, por lo que el asesoramiento y la organización son relevantes para el tema. González (2013) explica que el vínculo entre empresas trae muchos beneficios, para las pequeñas y medianas empresas (PYME), los vínculos son un medio para integrarse en la economía global y mejorar sus procesos de producción a través de tecnología, calidad y conocimientos gerenciales.

\section{CONCLUSIONES}

Posterior al análisis, se demuestra el gran potencial del turismo y que realmente puede ser un pilar clave para el desarrollo económico de Guanacaste. En los últimos años, la demanda de servicios turísticos ha aumentado y el aeropuerto de la zona ha evidenciado un importante aumento del tráfico turístico. Sobre el tema, se destacan conclusiones relacionadas a la situación actual de la exportación de servicios, las expectativas para los 
próximos años y los desafíos para promover la exportación de turismo y generar desarrollo.

En primer lugar, la situación actual de la exportación de servicios turísticos es muy positiva. Guanacaste se encuentra en una zona con grandes atractivos que generan ventajas competitivas como el aspecto ecológico que es clave; además, el ingreso de los turistas tiene un aumento considerable que comienza a superar los 3 millones en el año 2018. El turismo contribuye considerablemente a la economía del país y es un generador de empleo importante.

Como segundo punto, las expectativas del turismo en Guanacaste para los próximos años implican cambios importantes; los grandes proyectos empresariales y hoteleros y los aumentos en los permisos de construcción hacen soñar a la provincia, aunque se debe tener precaución ya que se requiere una preparación adecuada para poder enfrentar los avances que se presentan en aspectos tales como la infraestructura vial y los servicios.

En tercera conclusión, se deben reconocer los desafíos para la exportación de turismo para generar un desarrollo económico óptimo, implica la preparación del capital humano a través de la educación y evidentemente el idioma inglés. Además, es importante considerar una mayor colaboración entre las empresas para desarrollar vínculos y una mayor comunicación, de modo que más partes puedan proporcionar servicios turísticos. Para esto también es importante los esfuerzos del Gobierno y los municipios locales, así como las propias empresas, preparándose en productos y servicios de calidad, además de la apertura de relaciones rentables entre empresas.

Finalmente, el potencial del turismo es muy grande, pero no debería estar en unas pocas personas, las grandes empresas reciben turistas y entregan un paquete completo y las ganancias se dejan en pocas manos, por lo tanto, el ambiente de negocios debe mejorarse buscando que el turista visite muchas partes de Guanacaste y agregue valor a su visita, esto generará un impacto en más empresas y personas por su calidad de vida y, por lo tanto, el desarrollo económico. 
Para promover esto se deben establecer encadenamientos entre los diversos servicios turisticos, transportistas, empresas de restauración, hoteles, productores de insumos, entre otros, que deben coordinar actividades y reconocer que la unión y colaboración puede mejorar la experiencia del turista, además los gobiernos locales e instituciones deben buscar promover que la infraestructura en general mejore y así se facilite la creación y mejora de los atractivos turísticos y así lograr un destino cada vez más adaptable y competitivo.

\section{REFERENCIAS BIBLIOGRÁFICAS}

Área de Conservación Guanacaste (2017). ¿Qué es el Área de Conservación Guanacaste? Recuperado de https://www.acguanacaste.ac.cr/acg/que-es-el-acg

Barquero, M. (2017). Inversión en nuevos hoteles de Costa Rica ascenderá a \$149 millones en 2017. La Nación. Recuperado de http://www.nacion.com/economia/empresarial/Inversion-nuevos-hoteles-ascenderamillones 0 1613038692.html?utm source=rss\%3D\&utm medium=rss\%3D\&utm co ntent=2017-01-31\%3D\&utm campaign=rss-economia\%3D

Barquero, M. (2016). Costa Rica received 2,665,000 tourists in 2015. La Nación. Recuperado de http://www.nacion.com/economia/indicadores/turismo-ICT-ingresosturistas-Mauricio Ventura 0 1536646385.html

Instituto Costarricense de Turismo (2019a). Tourist figures. Disponible en http://www.ict.go.cr/es/estadisticas/cifras-turisticas.html

Instituto Costarricense de Turismo (2019b). Tourist Directory. Disponible en http://www.visitcostarica.com/ict/paginas/categoria. asp?idcate $=67 \& \mathrm{sPais}=14 \& \mathrm{cst}=1$

Fallas, C. (2017). Guanacaste se convierte en un imán para supermercados en Costa Rica. El financiero. Disponible en http://www.elfinancierocr.com/negocios/supermercados-Guanacaste-GessaWalmart-Auto Mercado-Megasuper 0 1124287580.html 
González, A. (2013). Las cadenas productivas. La Nación. Disponible en http://www.nacion.com/opinion/foros/encadenamientosproductivos 0 1381261892.html

Hernández, R., Méndez, S., Mendoza, C. y Cuevas, A. (2017). Fundamentos de Investigación. Ciudad de México: McGraw-Hill

Jiménez E. (2017). Aeropuerto internacional Daniel Oduber supera los 4 millones de pasajeros en los últimos cinco años. La Nación. Recuperado de http://www.nacion.com/economia/Ingreso-turistas-aeropuerto-Liberiaaumento 0 1609239113.html

Palafox Muñoz, A. (2013). El turismo como eje de acumulación. Nómadas. Critical Journal of Social and Juridical Sciences, 161-174.

Presidencia de la República De Costa Rica (2016). El turismo registra casi el $50 \%$ de las exportaciones de servicios del país. Recuperado de http://presidencia.go.cr/comunicados/2016/10/turismo-registra-casi-el-50-de-lasexportaciones-de-servicios-del-pais/

Rodríguez, A. (2017). Exportación de servicios será un 5\% más dinámica de lo esperado en 2016. El Financiero. Recuperado de http://www.elfinancierocr.com/economia-ypolitica/exportaciones-servicios-costa rica-comex-bccr 0 1099690021.html

Segura, G. (2017). El turismo genera 600 mil empleos directa e indirecta. La Prensa Libre. Recuperado de https:/www.laprensalibre.cr/Noticias/detalle/99761/turismogenera-600-mil-empleos-de-forma-directa-e-indirecta

Tîţu, M. A., Răulea, A. S., \& Tुîţu, Ş. (2016). Measuring service quality in tourism industry. Procedia-Social and Behavioral Sciences, 221, 294-301.

La Voz de Guanacaste (2016). We'll Say It Again: Speaking English Is Essential. The Voice of Guanacaste. Recuperado de 
http://www.vozdeguanacaste.com/es/articulos/2016/11/14/editorial-hay-que-decirlode-nuevo-hablar-ingles-es-determinante

World Tourism Organization (2017). Practical Guidelines for Integrated Quality Management in Tourism Destinations. Madrid: UNWTO Publication 
